\title{
Some constraints on the Yukawa parameters in the neutrino modification of the Standard Model $(\nu M S M)$ and CP-violation
}

\author{
Volodymyr M. Gorkavenko* and Stanislav I. Vilchynskiy ${ }^{\dagger}$ \\ Department of Physics, Taras Shevchenko National University of Kyiv, \\ 64 Volodymyrs'ka St., Kyiv, 01601, Ukraine
}

\begin{abstract}
The equations connecting elements of the Yukawa matrix to elements of the active neutrino mass matrix in the $\nu M S M$ theory (an extension of the Standard Model by a singlet of three right-handed neutrinos) was analyzed, and explicit relations for the ratio of the Yukawa matrix elements through elements of the active neutrino mass matrix were obtained. This relation can be used for getting more accurate constraints on the model parameters. Particularly, with the help of the obtained results we investigated CP-violating phase in the $\nu M S M$ theory. We demonstrate that even in the case when elements of the active neutrino mass matrix are real the baryon asymmetry can be generated also.
\end{abstract}

\section{Introduction}

The Standard Model (SM) [1] of the electroweak and the strong interactions is one of the greatest successes of the physics. It describes correctly the processes with participation of elementary particles to energy scale $\sim 100 \mathrm{GeV}$ and for individual processes to several $\mathrm{TeV}$. It predicted a number of particles, the overwhelming majority of which has been observed. However, it is well known that the SM does not account for several phenomena in particle physics, astrophysics and cosmology. Namely: the SM does not provide the dark matter candidate; the SM does not explain neutrino oscillations and the baryon asymmetry of the Universe; the SM can not solve the strong $\mathrm{CP}$ problem in particle physics, the primordial perturbations problem and the horizon problem in cosmology, etc.

The solutions of the above mentioned problems of the SM need some new physics between the electroweak and the Planck scales. An important challenge for the theoretical physics is to see if it is possible to solve them using only the extensions of the SM below the electroweak scale [2].

The SM is the renormalized theory which is based on $S U(3) \times S U(2) \times U(1)$ gauge group and contains three generations fermions. The left-handed components of fermions form

${ }^{*}$ E-mail: gorka@univ.kiev.ua

${ }^{\dagger}$ E-mail: sivil@univ.kiev.ua 
the weak isospin doublets relative to the $S U(2)$ group, and the right-handed components of all fermions except neutrinos are singlets of the weak isospin. The absence of the right-handed neutrino fields in the SM is due to the fact that neutrinos are considered as massless particles. However, the recent experimental discovery of the neutrino oscillation phenomenon [3, 4] (transitions between neutrinos of different flavors) is a proof that neutrinos have a nonzero mass. The current data show that the mass squared differences of active neutrino $\Delta m_{\text {atm }}^{2}=(2.5 \pm 0.2) \cdot 10^{-3} \mathrm{eV}^{2}$ and $\Delta m_{\text {sol }}^{2}=(8.0 \pm 0.3) \cdot 10^{-5} \mathrm{eV}^{2}$.

One of the simplest and the most promising ways of modification of the SM is an extension of the fermionic sector by adding the singlet of right-handed neutrinos, which do not take part in the SM gauge interaction: 1 . The consequence of the existence of two distinct scales $\Delta m_{\text {atm }}^{2}$ and $\Delta m_{\text {sol }}^{2}$ is that number of right-handed neutrinos must be greater than or equal to two. The introduction of only two right-handed neutrinos leads to the emergence of 11 new parameters in the modified theory, which may be able to explain the available experimental data on the oscillations of active neutrinos. In this case the model predicts the existence of two massive active neutrinos and one massless neutrino, which does not contradict the available experimental data. However, the extension of the SM by only two right-handed neutrinos does not solve the remaining problems of the SM, in particular, does not explain the nature of the dark matter [5, 6].

It turns out that introduction of three right-handed neutrinos with masses smaller than electroweak scale can provide explanations of the experimental data in particle physics, astrophysics, and cosmology. This model (called $\nu M S M)$ [5, 7] is the simplest extension 2 of the SM, which can explain simultaneously some of phenomena of the new physics without a new energy scale. Moreover, the parameters of the model (it contains 18 new parameters - three Majorana neutrino masses, three Dirac neutrino masses, six mixing angles and six CP-violating phases) can be chosen in such a way that the physics above the electroweak scale is not altered, while the following three phenomena beyond the SM are explained. a) All data on the neutrino oscillations can be fitted. The smallness of the neutrino masses is explained by the Type I of the "see-saw" mechanism [8]. b) Parameters of two heavier neutrinos can be chosen to allow baryogenesis. The masses of these particles can still be chosen below the electroweak scale. c) The lightest sterile neutrino can be intensively produced in the early universe and have cosmologically long life-time. So, it might be a viable dark matter candidate.

The topical problem is the determination of the parameters of the $\nu M S M$. One of the purpose of this work is to obtain restrictions on the values of the Yukawa's matrix elements from the $\nu M S M$ equations that connect elements of the Yukawa's matrix with elements of the active neutrinos mass matrix.

Since neutrinos in the SM are massless particles, the only source of CP-symmetry violation in weak interactions is a single complex element of the Kabbibo-KobayashiMaskawa matrix, which describes the mixing between the quarks of different generations. Due to the existence of neutrinos with nonzero masses it becomes possible to mix different generations of neutrinos. Moreover, in the $\nu M S M$ the mix between active and sterile neutrino is possible too. So, there is another possible source of generation of CP-symmetry

\footnotetext{
${ }^{1}$ This is why these neutrinos are called sterile neutrinos. The left-handed neutrinos of the SM are called active neutrinos.

${ }^{2}$ Various possibilities to incorporate neutrino masses in the theory are discussed, e.g., in [9].
} 
violation and the $\nu M S M$ theory has a much richer structure than the SM.

Another purpose of this work is to study $\mathrm{CP}$ violation in the frame of the $\nu M S M$ theory. As was shown in [5], effect of the CP violation on the baryogenesis in the $\nu M S M$ theory can be expressed through the CP-violating factor $\delta_{C P}$. This factor includes the above mentioned CP-violating phases. The baryon asymmetry is proportional to $\delta_{C P}$. An applicable expression for $\delta_{C P}$ was obtained in [11]. Investigation of this expression is the second goal of the work.

The paper is organized as follows. The first section contains basic relations and some results obtained in the $\nu M S M$. In the second section of the paper the $\nu M S M$ equations are analyzed in detail, and the explicit relations for ratio of the Yukawa matrix elements through the elements of active neutrino mass matrix are obtained. Using results of the second section the CP-violating factor is analyzed in the third section.

\section{The basic relations and some results of the $\nu M S M$}

In the $\nu M S M$ [5, 7] the following terms are added to the Lagrangian of the SM (without taking into account the kinetic terms):

$$
\begin{aligned}
\mathcal{L}^{a d}=-F_{\alpha I} \bar{L}_{\alpha} \tilde{\Phi} \nu_{I R}-\frac{M_{I J}}{2} \bar{\nu}_{I R}^{c} \nu_{J R}+\text { h.c. } & = \\
& =-\frac{h+v}{\sqrt{2}} \bar{\nu}_{\alpha L} F_{\alpha I} \nu_{I R}-\bar{\nu}_{I R}^{c} \frac{M_{I J}}{2} \nu_{J R}+\text { h.c. },
\end{aligned}
$$

where index $\alpha=e, \mu, \tau$ corresponds to the active neutrino flavors, indices $I, J$ run from 1 to $3, L_{\alpha}$ is for the lepton doublet, $\nu_{I R}$ the field functions of the sterile right-handed neutrinos, the superscript „c" means charge conjugation, $F_{\alpha I}$ is for the new (neutrino) matrix of the Yukawa constants, $M_{I J}$ for the Majorana mass matrix of the right-handed neutrinos, $\Phi$ for the field of the Higgs doublet in the unitary gauge, $\tilde{\Phi}=i \sigma_{2} \Phi^{*}, h$ is for the neutral Higgs field and the parameter $v$ determines minimum of the Higgs field potential $(v \cong 247 \mathrm{GeV})$.

In the SM the mass of fermions are generated due to interactions of fermions fields with scalar Higgs field. The structure of the SM is such that after spontaneous symmetry breaking the neutrino remains to be a massless particle 3 . The SM does not contain Dirac mass term $\sim \bar{\nu}_{L} \cdot \nu_{R}$ due to the absence in theory the right-handed neutrinos, and the Majorana mass term $\sim \bar{\nu}_{L}^{c} \cdot \nu_{L}$ is forbidden by $S U(2)_{L}$ invariance. The assumption about the existence of the right-handed neutrinos leads to the appearance of the Dirac as well as Majorana mass terms in the Lagrangian, which in the general case have the form (see, e.g., [12])

$$
\mathcal{L}^{D M}=-\left(\overline{\left(N_{L}\right)^{c}} \frac{M^{D M}}{2} N_{L}+\text { h.c. }\right)
$$

\footnotetext{
${ }^{3}$ There is a possibility to introduce the mass of neutrino after the electroweak symmetry breaking with help of the effective dimension 5 non-renormalizable operator $\frac{\lambda_{i j}}{\Lambda}\left(L_{i} \Phi\right)^{T}\left(L_{j} \Phi\right), i, j=e, \mu, \tau$, where $L$ is the $\mathrm{SU}(2)$ lepton doublets, $\Lambda$ is the cutoff high-energy scale [10. This operator breaks lepton number and can be obtained from (1.1) by integrating out heavy right-handed neutrinos.
} 
where

$$
N_{L}=\left(\begin{array}{c}
\nu_{L} \\
\nu_{R}^{c}
\end{array}\right) ; N_{L}^{c}=\left(\begin{array}{c}
\nu_{L}^{c} \\
\nu_{R}
\end{array}\right) ; M^{D M}=\left(\begin{array}{cc}
M_{L} & M_{D}^{T} \\
M_{D} & M_{R}
\end{array}\right)
$$

Comparing mass terms in (1.1) with (1.2) one can see

$$
M_{L}=0, \quad M_{D}=F^{+} \frac{v}{\sqrt{2}}, \quad M_{R}=M^{*},
$$

where $M, F$ are square matrix of the third order with elements $F_{\alpha I}$ and $M_{I J}$ (1.1).

As it was shown in [5, 7], the parameters of the $\nu M S M$ can be chosen in such a way to simultaneously explain the neutrino oscillations, the baryon asymmetry and to determine the nature of the dark matter. This requires the existence of two right-handed neutrinos with large practically the same masses $(\gtrsim 100 \mathrm{MeV})$ and one right-handed neutrino with a relatively small mass 4 .

In zero approximation the extended Lagrangian $\mathcal{L}_{\nu M S M}$ is assumed to be invariant under $U(1)_{e} \times U(1)_{\mu} \times U(1)_{\tau}$ transformations, that provides preservation of the $e, \mu, \tau$ lepton numbers separately as in the SM. In addition, it is assumed that two heavy sterile neutrinos interact with the active neutrinos, but the third (lightest) sterile neutrino does not interact5. This assumption can be realized by following matrix $M^{D M}$ [13]:

$$
M_{L}^{(0)}=0 ; \quad M_{R}^{(0)}=\left(\begin{array}{ccc}
0 & 0 & 0 \\
0 & 0 & M \\
0 & M & 0
\end{array}\right), \quad M_{D}^{(0)+}=\frac{v}{\sqrt{2}}\left(\begin{array}{ccc}
0 & h_{12} & 0 \\
0 & h_{22} & 0 \\
0 & h_{32} & 0
\end{array}\right)
$$

where $M$ is real and $h_{12}, h_{22}, h_{32}$ - are complex quantities.

In this approximation we have two massive right-handed neutrinos with equal mass $M$, the third right-handed neutrino is massless, and all active neutrinos have a zero mass, which is in contradiction with observable data. To adjust it next small terms are added to the matrix $M_{R}$ and $M_{D}$ [13]:

$$
M_{L}^{(1)}=0 ; \quad M_{R}^{(1)}=\left(\begin{array}{ccc}
m_{11} e^{-i \alpha} & m_{12} & m_{13} \\
m_{12} & m_{22} e^{-i \beta} & 0 \\
m_{13} & 0 & m_{33} e^{-i \gamma}
\end{array}\right) ; M_{D}^{(1)+}=\frac{v}{\sqrt{2}}\left(\begin{array}{ccc}
h_{11} & 0 & h_{13} \\
h_{21} & 0 & h_{23} \\
h_{31} & 0 & h_{33}
\end{array}\right)
$$

where $m_{i j}\left(m_{i j} \ll M\right)$ are real, but elements of first and second columns $\left(\left|h_{i 1}\right| \ll\left|h_{k 2}\right|\right.$, $\left.\left|h_{i 3}\right| \ll\left|h_{k 2}\right|\right)$ are in general complex elements.

These corrections break $U(1)_{e} \times U(1)_{\mu} \times U(1)_{\tau}$-symmetry, lead to the appearance of a small mass of the third right-handed neutrino, and remove the mass degeneracy for two heavy right-handed neutrinos. It ensures the appearance of extra small masses of the active neutrinos and nonzero mixing angles among them.

As is well known [12], the mass part of the Lagrangian (1.2) can be diagonalized by the transition from the basis of the gauge functions $N_{L}$ to the basis of the mass functions $n_{L}$ using an unitary matrix $V$, namely $N_{L}=V n_{L}$, so

$$
\bar{N}_{L}=\bar{n}_{L} V^{+} ; \quad N_{L}^{c}=\left(V^{+}\right)^{T} n_{L}^{c} ; \quad \bar{N}_{L}^{c}=\overline{\left(n_{L}\right)^{c}} V^{T}
$$

\footnotetext{
${ }^{4}$ For the time being the allowed region for the mass of the lightest sterile neutrino is $(1 \div 50) \mathrm{KeV}[2]$.

${ }^{5}$ The lightest neutrino is the dark matter candidate in the $\nu M S M$, just because it does not have to interact with other particles of the SM.
} 
where $V(6 \times 6)$ is a product of two matrices $V=W U$.

The $W$ matrix is introduced for the block diagonalization of the $M^{D M}$ matrix [14]. The explicit form of the $W$ matrix can be approximately found in the "see-saw" approach due to the smallness of matrix $\varepsilon=M_{R}^{-1} M_{D} \ll 1$ :

$$
W=\left(\begin{array}{cc}
1-\frac{1}{2} \varepsilon^{+} \varepsilon & \varepsilon^{+} \\
-\varepsilon & 1-\frac{1}{2} \varepsilon \varepsilon^{+}
\end{array}\right) .
$$

In this approximation the result of the block diagonalization has the form

$$
W^{T} M^{D M} W=\left(\begin{array}{cc}
-M_{D}^{T} M_{R}^{-1} M_{D} & 0 \\
0 & M_{R}
\end{array}\right)=\left(\begin{array}{cc}
M_{\text {light }} & 0 \\
0 & M_{\text {heavy }}
\end{array}\right),
$$

where matrices $M_{\text {light }}$ and $M_{\text {heavy }}$ are the mass matrices of the active and the sterile neutrinos correspondingly. Note that the elements of the matrix $M_{\text {light }}$ and accordingly the masses of the active neutrinos are completely determined by elements of the matrices $M_{D}$ and $M_{R}$.

The $U$ matrix has the form

$$
U=\left(\begin{array}{cc}
U_{1} & 0 \\
0 & U_{2}
\end{array}\right)
$$

where matrices $U_{(1,2)}$ (each of them are $(3 \times 3)$ matrix) are chosen for the diagonalization of block matrix $W^{T} M^{D M} W$

$$
\begin{aligned}
m=\operatorname{diag}\left(m_{1}, m_{2}, \ldots m_{6}\right)= & V^{T} M V= \\
& =U^{T} W^{T} M W U=\left(\begin{array}{cc}
U_{1}^{T} M_{\text {light }} U_{1} & 0 \\
0 & U_{2}^{T} M_{\text {heavy }} U_{2}
\end{array}\right) .
\end{aligned}
$$

There is a standard parametrization [3] for $U_{(1,2)}$ :

$$
\begin{aligned}
U_{(1,2)}=\left(\begin{array}{ccc}
c_{12} c_{13} & c_{13} s_{12} & s_{13} e^{-i \delta} \\
-s_{12} c_{23}-c_{12} s_{23} s_{13} e^{i \delta} & c_{12} c_{23}-s_{12} s_{23} s_{13} e^{i \delta} & s_{23} c_{13} \\
s_{12} s_{23}-c_{12} c_{23} s_{13} e^{i \delta} & -c_{12} s_{23}-s_{12} c_{23} s_{13} e^{i \delta} & c_{23} c_{13}
\end{array}\right) \times & \\
& \times\left(\begin{array}{ccc}
e^{i \alpha_{1} / 2} & 0 & 0 \\
0 & e^{i \alpha_{2} / 2} & 0 \\
0 & 0 & 1
\end{array}\right),
\end{aligned}
$$

where $c_{i j}=\cos \theta_{i j}, s_{i j}=\sin \theta_{i j}, \theta_{12}, \theta_{13}, \theta_{23}$ are the three mixing angles; $\delta$ is the Dirac phase, and $\alpha_{1}, \alpha_{2}$ are the Majorana phases. The angles $\theta_{i j}$ can be in the region $0 \leq \theta_{i j} \leq$ $\pi / 2$, phases $\delta, \alpha_{1}, \alpha_{2}$ vary from 0 to $2 \pi$. Each of the matrices $U_{(1)}$ and $U_{(2)}$ contains its own, independent angles and phases.

Thus, the determination of the masses of active and sterile neutrinos is reduced to the diagonalization of the matrix (1.9), where diagonalization can be carried out separately for the matrices $M_{\text {light }}$ and $M_{\text {heavy }}$. Since the matrix $M_{\text {light }}$ and $M_{\text {heavy }}$ are not Hermitian, it is more appropriate to find eigenvalues of Hermitian matrices $M_{\text {light }}^{+} M_{\text {light }}$ and $M_{\text {heavy }}^{+} M_{\text {heavy }}$ by solving corresponding equations. The found eigenvalues are the square of eigenvalues of the matrices $M_{\text {light }}$ and $M_{\text {heavy }}$. 
In the approximation when the elements of the first column of the Yukawa matrix are neglected and $M \gg m_{i j}$, the mass of the lightest active neutrino is zero. The nondiagonal mass matrix of the active neutrinos has the form [13]

$$
\left[M_{\text {light }}\right]_{\alpha \beta}=\eta\left(h_{\beta 3} h_{\alpha 2}+h_{\alpha 3} h_{\beta 2}\right), \quad \text { where } \quad \eta=v^{2} / 2 M
$$

and eigenvalues

$$
m_{2,3}=\eta\left(F_{2} F_{3} \pm\left|h^{+} h\right|_{23}\right),
$$

where $F_{2}^{2}=\left[h^{+} h\right]_{22}, F_{3}^{2}=\left[h^{+} h\right]_{33}, F_{2} F_{3}=M\left(m_{2}+m_{3}\right) / v^{2}$.

On the other hand, the elements of the matrix $M_{\text {light }}$ are defined by masses and mixing matrix $U_{(1)}$ of the active neutrinos [12]:

$$
\left[M_{\text {light }}\right]_{\alpha \beta}=m_{1} U_{(1) i 1}^{*} U_{(1) j 1}^{*}+m_{2} U_{(1) i 2}^{*} U_{(1) j 2}^{*}+m_{3} U_{(1) i 3}^{*} U_{(1) j 3}^{*} .
$$

The elements of the mixing matrix $U_{(1)}$ are known (unfortunately with a considerable inaccuracy) from the neutrino oscillation experiments (see, e.g., [3, 4]). Parameters of the matrix $U_{(1)}(1.12)$ are presented in Tab.1.

\begin{tabular}{cc}
\hline central value & $99 \%$ confidence interval \\
\hline$\left|\Delta m_{12}^{2}\right|=(8.0 \pm 0.3) \cdot 10^{-5} \mathrm{eV}^{2}$ & $(7.2-8.9) \cdot 10^{-5} \mathrm{eV}^{2}$ \\
$\left|\Delta m_{23}^{2}\right|=(2.5 \pm 0.2) \cdot 10^{-3} \mathrm{eV}^{2}$ & $(2.1-3.1) 10^{-3} \mathrm{eV}^{2}$ \\
$\tan ^{2} \theta_{12}=0.45 \pm 0.05$ & $30^{0}<\theta_{12}<38^{0}$ \\
$\sin ^{2} 2 \theta_{23}=1.02 \pm 0.04$ & $36^{0}<\theta_{23}<54^{0}$ \\
$\sin ^{2} 2 \theta_{13}=0 \pm 0.05$ & $\theta_{13}<10^{0}$ \\
\hline
\end{tabular}

Table 1. Experimental constraints on the parameters of active neutrinos.

\section{The ratio of the Yukawa matrix elements in the $\nu M S M$}

The system of equations (1.13) connects elements of the second and the third columns of the Yukawa sterile neutrinos matrix with elements of the active neutrinos matrix and has infinite number of solutions. Indeed, the replacement of $h_{i 2}$ to $z h_{i 2}$ and $h_{i 3}$ to $h_{i 3} / z$ ( $z$ is an arbitrary complex number) does not change the system (1.13).

But the system of equations (1.13) has a good solutions for the ratio of the Yukawa matrix elements. Indeed, if we take $h_{13}, h_{23}$, and $h_{33}$ from the equations for the diagonal elements of $M_{\text {light }}$ and substitute it into equations for the off-diagonal elements of $M_{\text {light }}$ we get

$$
\left\{\begin{array}{l}
M_{12}=\frac{1}{2}\left(M_{22} \frac{h_{12}}{h_{22}}+M_{11} \frac{h_{22}}{h_{12}}\right) \\
M_{13}=\frac{1}{2}\left(M_{33} \frac{h_{12}}{h_{32}}+M_{11} \frac{h_{32}}{h_{12}}\right) \\
M_{23}=\frac{1}{2}\left(M_{33} \frac{h_{22}}{h_{32}}+M_{22} \frac{h_{32}}{h_{22}}\right)
\end{array}\right.
$$


with the obvious solutions

$$
\left\{\begin{array}{l}
A_{12}=\frac{M_{12}}{M_{22}}\left(1 \pm \sqrt{1-\frac{M_{11} M_{22}}{M_{12}{ }^{2}}}\right) \\
A_{13}=\frac{M_{13}}{M_{33}}\left(1 \pm \sqrt{1-\frac{M_{11} M_{33}}{M_{13}^{2}}}\right) \\
A_{23}=\frac{M_{23}}{M_{33}}\left(1 \pm \sqrt{1-\frac{M_{22} M_{33}}{M_{23}}}\right)
\end{array}\right.
$$

for the ratio of the second column elements of the Yukawa matrix

$$
A_{12}=h_{12} / h_{22} ; \quad A_{13}=h_{12} / h_{32} ; \quad A_{23}=h_{22} / h_{32}
$$

The ratio of the third column elements of the Yukawa matrix can be easily obtained from the equations for the diagonal elements of $M_{\text {light }}$ :

$$
\frac{h_{23}}{h_{13}}=A_{12} \frac{M_{22}}{M_{11}} ; \quad \frac{h_{33}}{h_{13}}=A_{13} \frac{M_{33}}{M_{11}} .
$$

So, in the approximation when (1.13) is valid the ratio of the Yukawa matrix elements depends only on the active neutrino mass matrix.

Though formally there are eight different choices for the solutions, only four are independent. For example, if we fix the sign before the square roots in the expressions for $A_{12}$ and $A_{13}$ then $A_{23}$ is unambiguously determined by the relation

$$
A_{23}=A_{13} / A_{12}
$$

It should be emphasized that solutions (2.2) do not allow one to find elements of the Yukawa matrix but only its ratid:

$$
\begin{gathered}
\frac{\left\{h_{12} ; h_{22} ; h_{32}\right\}}{F_{2}}=\frac{e^{i \cdot \arg \left(h_{12}\right)}}{\sqrt{1+\left|A_{12}\right|^{-2}+\left|A_{13}\right|^{-2}}}\left\{1 ; A_{12}{ }^{-1} ; A_{13}{ }^{-1}\right\}, \\
\frac{\left\{h_{13} ; h_{23} ; h_{33}\right\}}{F_{3}}=\frac{e^{i \cdot \arg \left(h_{13}\right)}}{\sqrt{1+\left|A_{12} \frac{M_{22}}{M_{11}}\right|^{2}+\left|A_{13} \frac{M_{33}}{M_{11}}\right|^{2}}}\left\{1 ; A_{12} \frac{M_{22}}{M_{11}} ; A_{13} \frac{M_{33}}{M_{11}}\right\},
\end{gathered}
$$

where phases of $h_{12}, h_{13}$ are connected by condition

$$
\arg \left(h_{12}\right)+\arg \left(h_{13}\right)=\arg \left(M_{11}\right)
$$

and $A_{12}, A_{13}, M_{11}, M_{22}, M_{33}$ are definitely expressed via parameters of the active neutrino mass matrix. Since the system of equations (1.13) is written in the approximation $m_{1}=0$, the phase $\alpha_{1}$ is excluded from all expressions (see (1.12) and (1.15)). In this approximation

\footnotetext{
${ }^{6}$ It can be shown that our results (2.6), (2.7) coincide with results of [1] where the ratios of the elements were obtained in the particular case $\theta_{13} \rightarrow 0, \theta_{23} \rightarrow \pi / 4$.
} 
only seven parameters of active neutrinos are used: two mass $m_{2}, m_{3}$; three mixing angles $\theta_{12}, \theta_{13}, \theta_{23}$; one Dirac $\delta$ and one Majorana $\alpha_{2}$ CP-violating phases.

To know straight values of the Yukawa matrix elements we have to know in addition two arbitrary parameters 7 of Yukawa matrix, e.g., absolute value and phase of an arbitrary Yukawa matrix element or quantities $\xi$ and $\epsilon$ from (3.3).

It can be shown that (for fixed values of the active neutrino parameters) there are only two choices 8 for placing of the signs in the expressions for $A_{12}, A_{13}, A_{23}(2.2)$ which are not inconsistent with condition (2.5) . These two variants are distinguished from each other by simultaneous replacement of the sign in front of square roots in the expressions for $A_{12}, A_{13}, A_{23}$. It can be shown that such replacement of the signs leads to interchanging and conjugating of the relation between elements of the second and the third columns of the Yukawa matrix, notably $h_{22} / h_{12} \leftrightarrow h_{23}^{*} / h_{13}^{*}, h_{32} / h_{12} \leftrightarrow h_{33}^{*} / h_{13}^{*}$.

The solutions of (1.13) can be analyzed numerically. Really, using condition (2.5) for setting correct signs in (2.2), we can straightly find the ratio of elements of the second $\left(\left|h_{22} / h_{12}\right|=A_{12}^{-1} ;\left|h_{32} / h_{12}\right|=A_{13}^{-1}\right)$ and the third $\left(\left|h_{23} / h_{13}\right|=A_{12} \frac{M_{22}}{M_{11}} ;\left|h_{33} / h_{13}\right|=A_{13} \frac{M_{33}}{M_{11}}\right)$ columns of the Yukawa matrix for every fixed point in the space of values $m_{2}, m_{3}, \theta_{12}$, $\theta_{13}, \theta_{23}, \alpha_{2}, \delta$.

In the case of the normal hierarchy the masses of active neutrinos increase with increasing their numbers $\left(m_{1}<m_{2}<m_{3}\right)$. In the $\nu M S M m_{1}=0$, so we take central values $m_{2}=\sqrt{\left|\Delta m_{12}^{2}\right|}=0.009 \mathrm{eV}, m_{3}=\sqrt{\left|\Delta m_{23}^{2}\right|}=0.05 \mathrm{eV}$. The phases $\delta, \alpha_{2}$ vary in range from $-\pi$ to $\pi$, the angles $\theta_{12}, \theta_{13}, \theta_{23}$ vary in accordance with Tab.1. With help of the numerical analysis we found the minimum and the maximum values for the ratio of elements of the second column of the Yukawa matrix and obtained $0.65 \lesssim\left|h_{32} / h_{12}\right| \lesssim 24.2$, $1.4 \lesssim\left|h_{22} / h_{12}\right| \lesssim 29.6$. Also, we found values of $\left(\left|h_{22} / h_{12}\right| ;\left|h_{32} / h_{12}\right|\right)$ and $\left(\operatorname{Arg}\left[h_{22} / h_{12}\right]\right.$; $\left.\operatorname{Arg}\left[h_{32} / h_{12}\right]\right)$ for $10^{4}$ points in the space of values $\theta_{12}, \theta_{13}, \theta_{23}, \alpha_{2}, \delta$. Results of calculations are demonstrated in Fig.1.
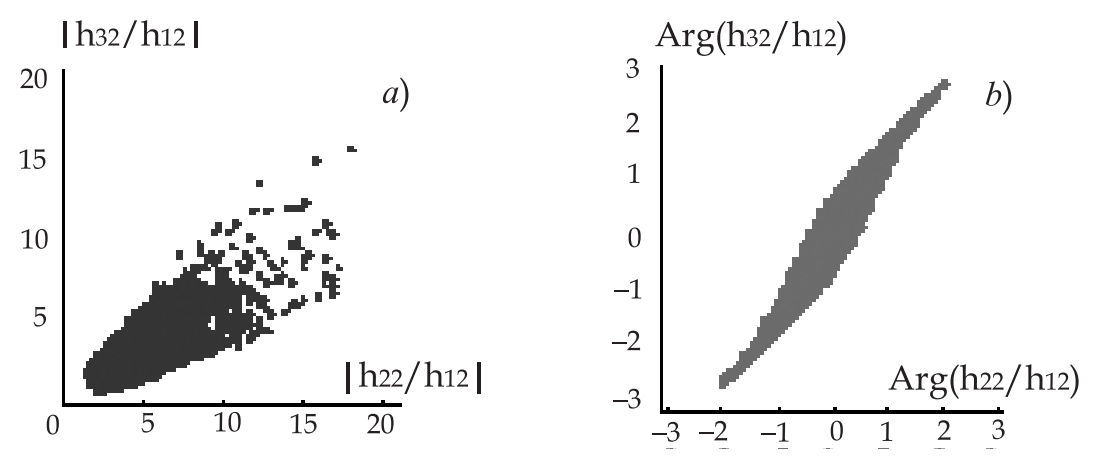

Fig. 1 The ratio of modules $a$ ) and difference of phases $b$ ) of the second column elements of the Yukawa matrix in the case of the normal hierarchy.

7 Only the relation between absolute values of the elements of the second and third columns of the Yukawa matrix $\epsilon=F_{3} / F_{2}$ was used as an additional relation in [13. It allows one to find out only the absolute values of the Yukawa's elements.

${ }^{8}$ This assertion is always true, except the special case of the parameters when at least one radical expression in (2.2) is zero. 
It should be noted that if one arbitrary takes a point in the space of values $\theta_{12}$, $\theta_{13}, \theta_{23}, \alpha_{2}, \delta$ there is a strong probability that $\left|h_{32} / h_{12}\right|$ and $\left|h_{22} / h_{12}\right|$ will lie in range from 1 to 10 and it is improbable that ratio of the Yukawa couplings will be greater than ten. The values of the phase differences $\operatorname{Arg}\left[h_{32} / h_{12}\right]$ and $\operatorname{Arg}\left[h_{22} / h_{12}\right]$ lie not in full region $[-\pi, \pi]$ but only in a closed compact region (Fig. $1, b$ ).

In the case of the inverse hierarchy the masses of active neutrinos increase with reducing their numbers $\left(m_{1}>m_{2}>m_{3}\right)$ but the $\nu M S M$ predicts $m_{1}=0$. In order to conform it we can swap the mass state $m_{3}$ and $m_{1}$ with help of additional rotation via unitary anti-diagonal matrix $\tilde{U}: U_{(1)} \rightarrow U_{(1)} \tilde{U}$ where $U_{(1)}$ is the mixing matrix of the active neutrinos (1.12). Assuming $m_{1}=0$, we get central values $m_{2}=m_{3}=\sqrt{\left|\Delta m_{23}^{2}\right|}=0.05 \mathrm{eV}$ and found the same quantities for the same range of values $\theta_{12}, \theta_{13}, \theta_{23}, \alpha_{2}, \delta$ like in the case of normal hierarchy. In this case the ratio of elements of the second column of the Yukawa matrix lies in range $0 \leq\left|h_{32} / h_{12}\right| \lesssim 3.2,1.1 \lesssim\left|h_{22} / h_{12}\right| \lesssim 4.3$. The points in the space of values $\theta_{12}, \theta_{13}, \theta_{23}, \alpha_{2}, \delta$ are demonstrated by Fig. 2 .

In this case the boundary great values of the elements ratio are improbable too. The ratio $\left|h_{32} / h_{12}\right|$ can be equal to zero because of $\left|h_{32} / h_{12}\right|=A_{12}^{-1} \sim M_{12}$ and $M_{12}$ can be equal zero in allowed range of parameters (Tab.1) under condition $m_{1}=m_{2}$. So, in contrast to the case of the normal hierarchy, elements $\left|h_{i 2}\right|$ can be of different order of magnitude. Similarly to the case of the normal hierarchy, the phase differences $\operatorname{Arg}\left[h_{32} / h_{12}\right]$ and $\operatorname{Arg}\left[h_{22} / h_{12}\right]$ lies in closed compact region (Fig.2,b).
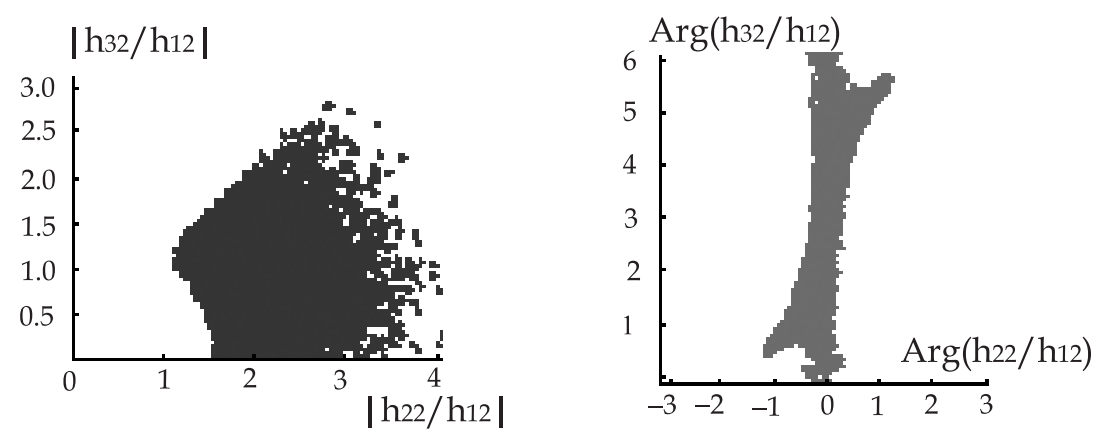

Fig.2 The ratio of modules $a$ ) and difference of phases $b$ ) of the second column elements of the Yukawa matrix in the case of the inverse hierarchy.

In the case of normal and inverse hierarchies the graphical representation of ratio of the third column elements $\left(\left|h_{33} / h_{13}\right| ;\left|h_{23} / h_{13}\right|\right)$ is identical to Fig.1,2(a). The corresponding representation of the phase differences $\left(\operatorname{Arg}\left[h_{33} / h_{13}\right] ; \operatorname{Arg}\left[h_{23} / h_{13}\right]\right)$ is identical to Fig. $1,2(b)$ but in the region $[0 ; 2 \pi]$.

The obtained constraints on the possible values of ratio of the Yukawa sterile neutrinos matrix elements are determined by the current data on active neutrino parameters. When the inaccuracy of the active neutrino parameters will decrease the constraints will be improved too. Obtained results can be useful for getting more accurate predictions of the $\nu M S M$ theory particularly for the investigation of the baryon asymmetry.

As one can see, there are four independent variants of solutions of (1.13). It should be noted that even in the case when the masses and mixture angles of active neutrinos are known exactly we will be not able to say which of these variants of the solutions are 
realized. To known it we need the values of phases of the active neutrinos matrix $U_{1}$. For fixed values of the all active neutrino matrix parameters the quantity of the possible solutions reduces to two that corresponds to interchanging and conjugating of the relation between elements of the second and the third columns of the Yukawa matrix. It is closely related to the symmetry of (1.13) under replacing the elements of the second column of the Yukawa matrix by elements of the third column. In this case two variants of solutions in principle can be distinguished experimentally with help of measurement of the mixture angles between active and sterile neutrinos.

\section{CP-violation in the $\nu M S M$}

As is known [15], in order that the baryon asymmetry generated from the initial charge symmetric state of the hot Universe, the next conditions must be satisfied simultaneously: a) baryon number non-conservation; b) $\mathrm{C}$ - and CP-violation; c) deviations from thermal equilibrium.

In the field theory the Lagrangian under CP-transformations turns into the Lagrangian with complex conjugated couplings. If theory contains couplings with unremovable phases, then this theory is not CP-invariant. The unique source of CP-violation in the SM is one complex element in the Cabibbo-Kobayashi-Maskawa mixing matrix of quarks. In the $\nu M S M$ theory, owing to massiveness of neutrinos, there is mixing among different generations of neutrinos and therefore there is additional possible source of CP-symmetry violation. This theory has a possibility of the baryon asymmetry generation due to existence of CP-violating oscillations of active neutrinos into sterile. Such oscillations change the full lepton number of a system and create lepton asymmetry that transforms into the baryon asymmetry with the help of the electroweak sphalerons [2, 16].

To analyze CP-symmetry violation in the $\nu M S M$ we use relation for the $\mathrm{CP}$-violating factor [11]

$$
\delta_{C P}=\frac{1}{F_{2}^{6}}\left[\operatorname{Im}\left(h^{+} h\right)_{23} \sum_{\alpha}\left(\left|h_{\alpha 2}\right|^{4}-\left|h_{\alpha 3}\right|^{4}\right)-\left(F_{2}^{2}-F_{3}^{2}\right) \sum_{\alpha}\left(\left|h_{\alpha 2}\right|^{2}+\left|h_{\alpha 3}\right|^{2}\right) \operatorname{Im}\left[h_{\alpha 2}^{*} h_{\alpha 3}\right]\right] .
$$

The solutions (2.2) allow us to express the last relation through the parameters of the active neutrinos. We get

$$
\begin{aligned}
\delta_{C P}(\xi, \varepsilon)=\left|M_{11}\right|^{-1} C^{-3}\left[\varepsilon\left(\operatorname{Im}\left[e^{-2 i \xi} A\right] B-C D\right)\right. & +\varepsilon^{3}\left(C_{1} D-C D_{1}\right)+ \\
& \left.+\varepsilon^{5}\left(C_{1} D_{1}-B_{1} \operatorname{Im}\left[e^{-2 i \xi} A\right]\right)\right],
\end{aligned}
$$

where there is dependence on only two parameters of the Yukawa matrix

$$
\xi=\arg \left[h_{12}\right], \quad \varepsilon=\left|h_{13} / h_{12}\right|=\epsilon \sqrt{C / C_{1}},
$$


and following notations are used:

$$
\begin{aligned}
& \epsilon=F_{3} / F_{2} ; A=M_{11}+\frac{A_{12}}{A_{12}^{*}} M_{22}+\frac{A_{13}}{A_{13}^{*}} M_{33} ; \\
& B=1+\left|A_{12}\right|^{-4}+\left|A_{13}\right|^{-4} ; \quad C=1+\left|A_{12}\right|^{-2}+\left|A_{13}\right|^{-2} ; \\
& B_{1}=1+\left|A_{12} \frac{M_{22}}{M_{11}}\right|^{4}+\left|A_{13} \frac{M_{33}}{M_{11}}\right|^{4} ; \quad C_{1}=1+\left|A_{12} \frac{M_{22}}{M_{11}}\right|^{2}+\left|A_{13} \frac{M_{33}}{M_{11}}\right|^{2} ; \\
& D=\operatorname{Im}\left[e^{-2 i \xi} M_{11}\right]+\left|A_{12}\right|^{-2} \operatorname{Im}\left[e^{-2 i \xi} \frac{A_{12}}{A_{12}^{*}} M_{22}\right]+\left|A_{13}\right|^{-2} \operatorname{Im}\left[e^{-2 i \xi} \frac{A_{13}}{A_{13}^{*}} M_{33}\right] ; \\
& D_{1}=\operatorname{Im}\left[e^{-2 i \xi} M_{11}\right]+\left|\frac{M_{22}}{M_{11}} A_{12}\right|^{2} \operatorname{Im}\left[e^{-2 i \xi} \frac{A_{12}}{A_{12}^{*}} M_{22}\right]+\left|\frac{M_{33}}{M_{11}} A_{13}\right|^{2} \operatorname{Im}\left[e^{-2 i \xi} \frac{A_{13}}{A_{13}^{*}} M_{33}\right] .
\end{aligned}
$$

Numerical analysis of the relation (3.2) confirms the general properties of the CPviolating factor (3.1) obtained in [11]:

1) sign of the CP-violating factor and correspondingly the sign of the baryon asymmetry can not be determined by only the elements of the active neutrino matrix;

2) if $\epsilon \rightarrow 0$, then $\delta_{C P} \sim \epsilon$ and also tends to zero;

3 ) the CP-violating factor can not be equal zero 9 when $\epsilon=1$;

4) the CP-violating factor can not be equal zero ${ }^{9}$ when $\theta_{13}=0$ and $\theta_{23}=\pi / 4$;

$5)$ in the case of inverse hierarchy the CP-violating factor can not be equal zero ${ }^{9}$ when $m_{1}=m_{2}, \theta_{13}=0, \theta_{23}=\pi / 4$.

The range of values of all parameters in the relation (3.2) is known 10 . This allows one to estimate bounds of possible values of the CP-violating factor. For the case of normal hierarchy we have $\left|\delta_{C P}\right| \lesssim 0.27$, for the case of inverse $-\left|\delta_{C P}\right| \lesssim 0.08$.

As one can see from (3.1) the CP-violating factor may be nonzero only in the case when Yukawa matrix elements have an imaginary part. The solutions (2.2) allow one to consider the possibility of the baryon asymmetry generation in the case when mixing matrix $U_{(1)}$ and, correspondingly to (1.15), the mass matrix of the active neutrino $M_{\text {light }}$ are real. It is easy to see that for real matrix $U_{(1)}(1.12)$ the following minors of the matrix $M_{\text {light }}=U_{(1)}^{*} m U_{(1)}^{+}$(here $\left.m=\operatorname{diag}\left(0, m_{2}, m_{3}\right)\right)$ are not negative:

$$
\left\{\begin{array}{l}
M_{11} M_{22}-M_{12}^{2}=m_{2} m_{3}\left(\sin \theta_{13} \cos \theta_{12} \cos \theta_{23}-\sin \theta_{12} \sin \theta_{23}\right)^{2} \geq 0 \\
M_{11} M_{33}-M_{13}^{2}=m_{2} m_{3}\left(\sin \theta_{13} \cos \theta_{12} \sin \theta_{23}+\sin \theta_{12} \cos \theta_{23}\right)^{2} \geq 0 \\
M_{22} M_{33}-M_{23}^{2}=m_{2} m_{3} \cos ^{2} \theta_{13} \cos ^{2} \theta_{12} \geq 0
\end{array}\right.
$$

If the values of the mixing angles are defined by Tab.1 all the above minors are positive. In this case the ratio of Yukawa's elements (2.3), (2.4) and, consequently, the Yukawa's elements are complex numbers. So, we can expect that CP-violating factor can be nonzero.

The direct numerical analysis of the CP-violating factor (3.2) confirms this assumption. Furthermore, if one takes arbitrary a point in the space of values $\theta_{12}, \theta_{13}, \theta_{23}, \xi, \varepsilon(\delta=$ $\left.\alpha_{2}=0\right)$ there is a strong probability that the CP-violating factor will be nonzerd 11 . Thus,

\footnotetext{
${ }^{9}$ For some particular values of mixing angles and phases $\delta_{C P}$ may be zero. This values of the parameters can be found numerically.

${ }^{10}$ The bounds of values of the mixing angles are defined by Tab.1, the phases are in the region $[0,2 \pi]$, $7 \cdot 10^{-5}<\epsilon<1[1]$.

${ }^{11}$ The equation for zero CP-violating factor (3.2) has a fine-tuning solutions in space of the mentioned parameters.
} 
even in the case when active neutrino mass matrix $M_{\text {light }}$ is real the electroweak generation of the baryon asymmetry can be realized also.

The CP-violating factor is a complicated function of parameters of the active neutrino matrix and the Yukawa matrix. The analysis of this factor is accentuated by fact that for fixed point in space of active neutrino parameters one needs to take appropriate variant of solutions of equations (1.13). The investigation of manifestation of the different variants of solutions (2.2) on the CP-violating factor is an interesting task for future.

\section{Conclusions}

The $\nu M S M$ is the minimal neutrino modification of the SM that can explain simultaneously neutrino oscillations, generation of the baryon asymmetry, and the nature of dark matter. There are strong conditions on the parameters of the $\nu M S M$ that can be experimentally checked. For the time being observable data, obtained from the missions XMM-Newton, Chandra, INTEGRAL, Suzaku, reveal no signs of existence of the sterile neutrino in predicted by the $\nu M S M$ and instrumentally allowed region (see [2] and references there). But new investigations are planed (for example, project Xenia [17]) that will continue to inspect theoretically allowed region of the model parameters.

Obtained in this paper exact solutions of the $\nu M S M$ equations connect elements of the Yukawa matrix with elements of the active neutrino mass matrix and will be useful for analysis, data processing, and getting more accurate constraints on the model parameters.

The analysis of the ratio of the Yukawa matrix elements demonstrates that in the case of normal hierarchy elements of second $\left(h_{i 2}\right)$ and third $\left(h_{i 3}\right)$ columns are the same order of magnitude. But in the case of inverse hierarchy the magnitudes of elements can considerably vary from each other in the column.

$\mathrm{CP}$-violating phase in the $\mathrm{SM}$ is parameter of the Cabibbo-Kobayashi-Maskawa matrix and it is known from observable data. In contrast to it the CP-violating factor $\left(\delta_{C P}\right)$ in the $\nu M S M$ is effective parameter that is present in the expression for the baryon asymmetry. Therefore the CP-violating factor in the $\nu M S M$ has a sophisticated structure as a function of the Yukawa matrix parameters.

Obtained solutions (2.2) allow one to get expression for the CP-violating factor (3.1) through the parameters of the active neutrinos and two parameters of the Yukawa matrix. Due to this the bounds of possible values of the CP-violating factor were estimated. It should be noted that in the case of the inverse hierarchy the maximum of $\left|\delta_{C P}\right|$ is considerably smaller (fourfold) as compared to the case of normal hierarchy.

As is known, the phases of the active neutrino mixing matrix can not be measured for the time being. It was shown that in any case (even if these phases are zero) the CP-violating factor can be nonzero and the baryon asymmetry generation is possible. The fact of the matter is that the elements of the Yukawa matrix are complex when the elements of the active neutrino mixing matrix are real.

As it was mentioned above the $\nu M S M$ provides a candidate for dark matter particle with mass $M_{1}$ in the range $(1 \div 50) \mathrm{KeV}$. It is the lightest sterile neutrino that is produced due to the resonant active-sterile neutrino oscillations in the presence of lepton asymmetry [11, 18]. It requires the high mass degeneracy of two other heavier neutrinos and leads to the fine-turning problem [11, 19]. 
It should be noted that modifications of the $\nu M S M$ can provide other production mechanisms of the lightest sterile neutrino due to interactions with other new particles [20] or primordial Higgs-inflation [21]. It applies some additional constraints on the parameters of the $\nu M S M$ (see, also, [19]) and changes the mass range of the lightest sterile neutrino. The change of only $M_{1}$ has no action on the results of the present work but the additional terms in the Lagrangians of such theories can modify the equations (1.13) that should be taken into account for investigations of the constraints in such theories.

We would like to thank A. Boyarsky, O. Ruchayskiy, D. Iakubovskyi and M. Shaposhnikov for the idea of treating this subject, and for useful comments and discussions. This work has been supported by the Swiss Science Foundation (grant SCOPES 2010-2012, No. IZ73Z0_128040).

The final publication is available at www.epj.org

\section{References}

[1] S. Weinberg, Phys. Rev. Lett. 19 (1967) 1264; S. L. Glashow, Nucl. Phys. 22 (1961) 579; A. Salam, Proceedings Of The Nobel Symposium Held 1968 At Lerum, Sweden, Stockholm 1968, 367-377.

[2] A. Boyarsky, O. Ruchayskiy, and M. Shaposhnikov, Ann. Rev. Nucl. Part. Sci. 59, $191(2009)$.

[3] Particle Data Group, http://pdg.lbl.gov

[4] A. Strumia and F. Vissani, arXiv: hep-ph/0606054.

[5] T. Asaka, S. Blanchet, and M. Shaposhnikov, Phys. Let. B 631, 151 (2005).

[6] M. Shaposhnikov, Prog. Theor. Phys., 122, No. 1, 185 (2009).

[7] T. Asaka and M. Shaposhnikov, Phys. Let. B 620, 17 (2005).

[8] R.N. Mohapatra and A.Y. Smirnov, Ann.Rev.Nucl.Sci.56, 569 (2006).

[9] S.F. King, Rept. Prog. Phys. 67, 107 (2004); G. Altarelli and F. Feruglio, New J. Phys. 6, 106 (2004).

[10] S. Weinberg, Phys. Rev. Lett. 43, 1566 (1979).

[11] M. Shaposhnikov, arXiv:0804.4542.

[12] S. Bilenky and S. Petcov, Rev. Mod. Phys. 59 No. 3, Part 1 (1987).

[13] M. Shaposhnikov, Nucl. Phys. B 763, 49 (2007).

[14] S. Bilenky, C. Giunti, and W. Grimus, Prog. Part. Nucl. Phys. 43, 1 (1999).

[15] A.D. Sakharov, JETP Lett. 5, 24 (1967). 
[16] E.K. Akhmedov, V.A. Rubakov, and A.Y. Smirnov, Phys. Rev. Lett. 81, 1359 (1998); V.A. Kuzmin, V.A. Rubakov, and M.E. Shaposhnikov, Phys. Lett. B 155, 36 (1985); V.A. Kuzmin, V.A. Rubakov, and M.E. Shaposhnikov, Phys. Lett. B 191, 171 (1987); S.Y. Khlebnikov and M.E. Shaposhnikov, Phys. Lett. B 387, 817 (1996).

[17] J.W. den Herder et al., arXiv:0906.1788.

[18] X. Shi, G.M. Fuller, Phys. Rev. Lett. 82, 2832 (1999).

[19] A. Roy, M. Shaposhnikov, arXiv:1006.4008.

[20] M. Shaposhnikov and I. Tkachev, Phys. Lett. B 639, 414 (2006); A. Kusenko, Phys. Rev. Lett. 97, 241301 (2006); A. Anisimov, Y. Bartocci, and F. L. Bezrukov, Phys. Lett. B 671, 211 (2009); F. Bezrukov and D. Gorbunov, JHEP 05, 010 (2010).

[21] F. Bezrukov, D. Gorbunov, and M. Shaposhnikov, JCAP 0906, 029 (2009). 